volume 9

ISSUE 5

2021 dicembre

Editor in Chief Michele Meschi

Supervisor Editor Roberto Nardi

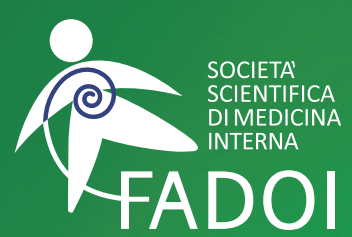

FEDERAZIONE

DELLE ASSOCIAZIONI

DEI DIRIGENTI

OSPEDALIERI

INTERNISTI

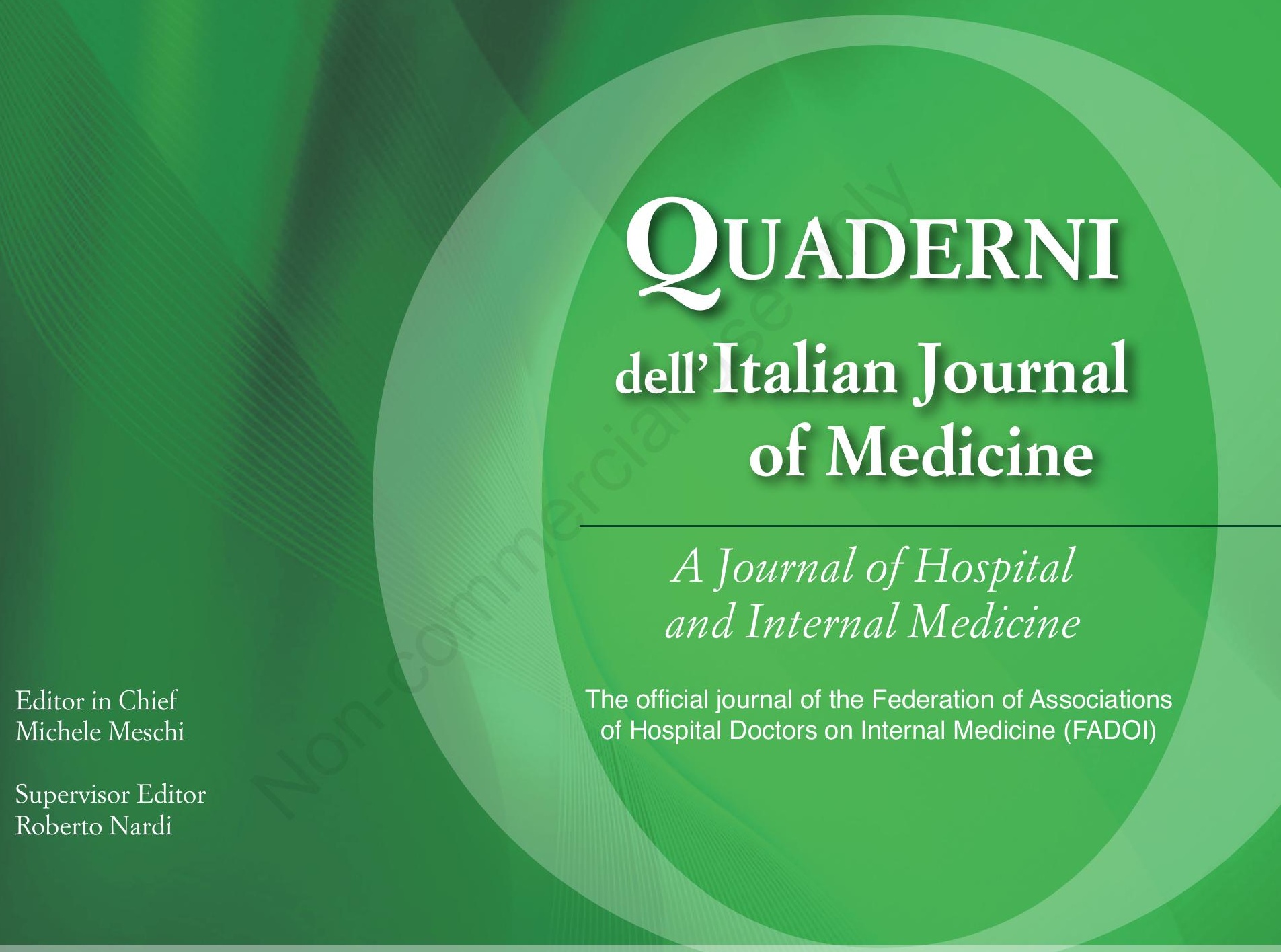

Considerazioni condivise alla luce delle recenti novità nell'ambito dello scompenso cardiaco: ottimizzazione della gestione del paziente con scompenso cardiaco in Medicina Interna - dal ricovero al follow-up

Board editoriale: F. Dentali, G. Campagna, M. Cavalleri, F. Gallucci, F. Pieralli 


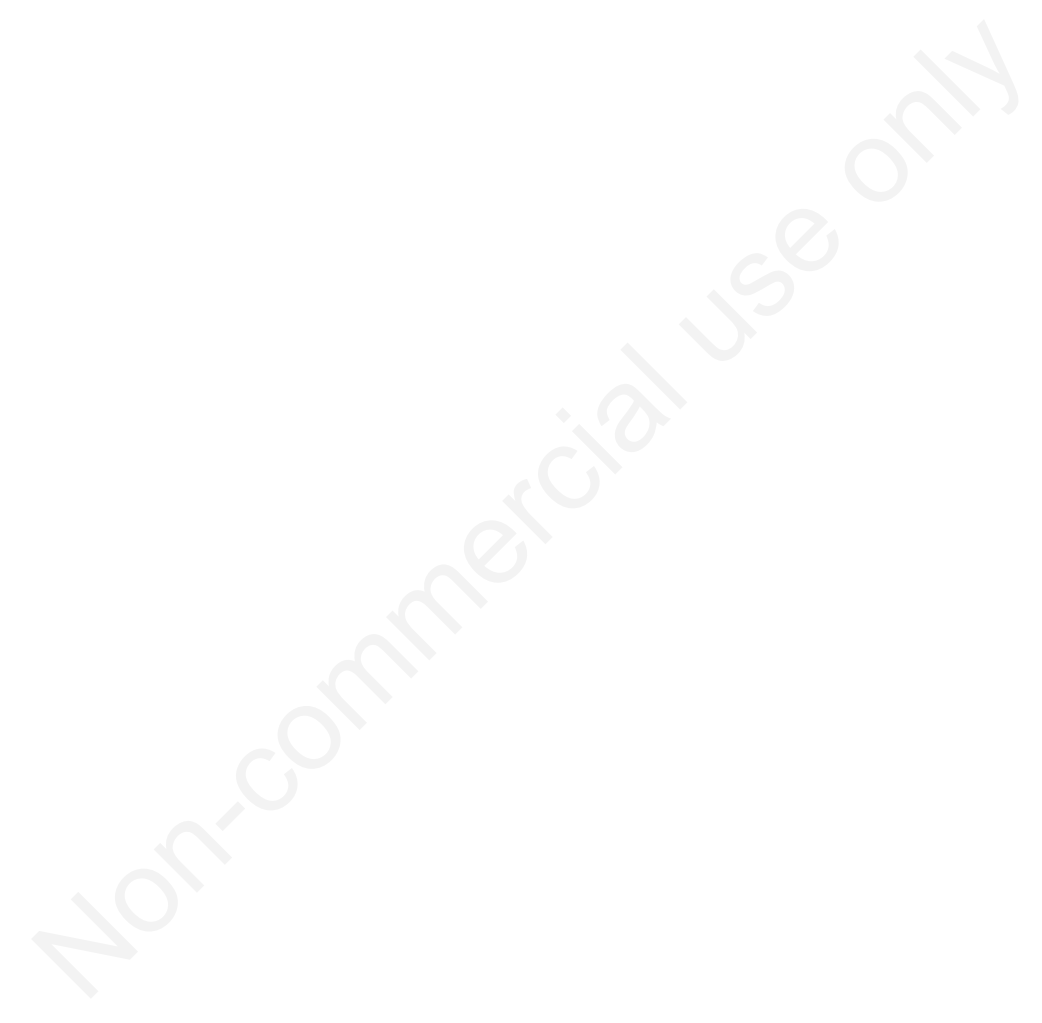


Via Felice Grossi Gondi, 49 - 00162 Roma Tel. +39.06.85355188 - Fax: +39.06.85345986 E-mail: segreteria@fadoi.org

\section{Società Scientifica FADOI - Organigramma}

PRESIDENTE NAZIONALE

\section{Dario Manfellotto, Roma, Italy}

PRESIDENTE ELETTO

Francesco Dentali, Varese, Italy

PAST PRESIDENT

Andrea Fontanella, Napoli, Italy

SEGRETARIO

Paola Gnerre, Savona, Italy

\section{STAFF DI SEGRETERIA}

Lorenza Lenzi, Pomarolo (TN), Italy

Ada Maffettone, Napoli, Italy

Claudia Tieri, Bari, Italy

TESORIERE

Giorgio Ballardini, Rimini, Italy

COORDINATORE COMMISSIONE GIOVANI

Ombretta Para, Firenze, Italy

RESPONSABILE RAPPORTI ISTITUZIONALI

Claudio Santini, Roma, Italy

RESPONSABILE RAPPORTI CON LE REGIONI

Alberto Fortini, Firenze, Italy

RESPONSABILE EVENTI E INIZIATIVE SPECIALI

Mauro Campanini, Novara, Italy

EDITOR IN CHIEF ITALIAN JOURNAL OF MEDICINE

Giorgio Vescovo, Padova, Italy
RESPONSABILE DEI

QUADERNI DELL'ITALIAN JOURNAL OF MEDICINE EDIZIONI ON LINE

Michele Meschi, Borgo Val di Taro (PR), Italy

SUPERVISOR EDITOR DEI

QUADERNI DELL'ITALIAN JOURNAL OF MEDICINE

Roberto Nardi, Bologna, Italy

RESPONSABILE SITO WEB E COMUNICAZIONE SOCIAL

Salvatore Lenti, Arezzo, Italy

Giuseppe Oteri, Milano, Italy

WEB MANAGER E CONTENT EDITOR

Giuseppe Oteri, Milano, Italy

Davide Ghilardi, Milano, Italy

RESPONSABILE SISTEMA GESTIONE QUALITÀ

Franco Berti, Roma, Italy

DPO

Alba Sciascera, Magenta (MI), Italy

\section{CONSULTA DEI PRESIDENTI}

Sandro Fontana, Biella, Italy

Salvatore Di Rosa, Palermo, Italy

Ido Iori, Reggio Emilia, Italy

Antonino Mazzone, Legnano (MI), Italy

Carlo Nozzoli, Firenze, Italy

Mauro Campanini, Novara, Italy

Andrea Fontanella, Napoli, Italy 


\section{COORDINATORE}

David Terracina, Roma, Italy

\section{SEGRETARIO E RESPONSABILE SCIENTIFICO} DEL PROVIDER ECM

Andrea Montagnani, Grosseto, Italy

DIRETTORI DEL DIPARTIMENTO

DELLA RICERCA CLINICA FADOI

Filippo Pieralli, Firenze, Italy

Fulvio Pomero, Savigliano (CN), Italy

DIRETTORI DIPARTIMENTO

PER LA FORMAZIONE E AGGIORNAMENTO

Luigi Magnani, Voghera (PV), Italy

Roberta Re, Novara, Italy

\section{COORDINATORE SCIENTIFICO CENTRO STUDI FONDAZIONE FADOI}

Gualberto Gussoni, Milano, Italy

\section{DELEGATI SOCIETÀ SCIENTIFICHE COLLEGATE}

FISM

SIF

Consulta cardiovascolare

SIIA e ISO

Antonino Mazzone, Legnano (MI), Italy

Luigi Magnani, Voghera (PV), Italy

Michele Stornello, Siracusa, Italy

Michele Stornello, Siracusa, Italy

Arcangelo Iannuzzi, Pomigliano d'Arco (NA), Italy

EFIM

Antonio Brucato, Milano, Italy

Lorenza Lenzi, Pomarolo (TN), Italy

Choosing wisely Roberto Frediani, Chieri (TO), Italy

\section{PROGETTI SPECIALI}

AGGIORN@FADOI

Giuliano Pinna

PROGETTO NUOVE TECNOLOGIE

Francesco Nasso, Flavio Tangianu

PROGETTO MEDICINA DI GENERE

Cecilia Politi

PROGETTO COMPETENCE

Flavio Tangianu

PROGETTO GOVERNANCE

Stefano De Carli, Andrea Montagnani, Fabrizio Colombo

PROGETTO HOSPITALIST

Francesco Orlandini

PROGETTO FINE VITA

Mauro Carbone, Fabio Gilioli

PROGETTO GASTROENTEROLOGIA e FEGATO

Luca Fontanella, Paola Piccolo, Franco Radaelli, Giancarlo Parisi

PROGETTO NUTRIZIONE CLINICA

Roberto Risicato, Luciano Tramontano

PROGETTO MALATTIE INFETTIVE/ANTIBIOTICI

Claudio Santini, Massimo Giusti, Marco Falcone

PROGETTO ECOGRAFIA INTERNISTICA

Francesco Cipollini, Nicola Mumoli

PROGETTO MALATTIE RARE

Antonio Brucato, Antonella Paradiso

PROGETTO BPCO/NIV

Marco Candela, Giuseppe De Matthaeis, Francesco Ventrella

PROGETTO TROMBOSI

Mauro Silingardi, Matteo Giorgi Pierfranceschi, Pierpaolo Di Micco

PROGETTO TRIAL

Giancarlo Agnelli, Antonio Ceriello, Leo Fabbri, Claudio Ferri,

Franco Radaelli, Paolo Verdecchia

\section{PRESIDENTE ANÍMO}

Gabriella Bordin, Castelfranco Veneto (TV), Italy 


\title{
QUADERNI - Italian Journal of Medicine
}

\author{
CONSIDERAZIONI CONDIVISE ALLA LUCE DELLE RECENTI \\ NOVITÀ NELL'AMBITO DELLO SCOMPENSO CARDIACO: \\ OTTIMIZZAZIONE DELLA GESTIONE DEL PAZIENTE \\ CON SCOMPENSO CARDIACO IN MEDICINA INTERNA - \\ DAL RICOVERO AL FOLLOW-UP
}

Board editoriale:

Francesco Dentali, Ospedale Fondazione Macchi di Varese - Coordinatore Giuseppe Campagna, Ospedale Santa Maria Goretti di Latina Maurizio Cavalleri, ASL 4 PO Sestri Levante Fernando Gallucci, Ospedale Cardarelli di Napoli Filippo Pieralli, Ospedale Universitario Careggi di Firenze

Nuova classificazione dello scompenso cardiaco: ridenominazione di Heart failure with mid-range ejection fraction in Heart failure with mildly reduced ejection fraction (HFmrEF), con raccomandazioni terapeutiche più precise in questa fascia di pazienti .

Il nuovo algoritmo per il trattamento dello scompenso cardiaco a frazione di eiezione ridotta: Simultaneous or Rapid Sequence Initiation of Quadruple Medical Therapy for Heart Failure

Ottimizzazione della terapia: l'ospedalizzazione come opportunità.

Impiego precoce di sacubitril/valsartan nel paziente ospedalizzato, stabilizzato. Effetti sulla riduzione della mortalità e delle re-ospedalizzazioni.

Effetti sul rimodellamento cardiaco.

Effetti sulla QoL

Sicurezza dell'uso di sacubitril/valsartan anche in presenza di comorbidità e gestione dell'ipotensione

Intervento di ottimizzazione della terapia anche nel paziente con scompenso cardiaco cronico ricoverato per altra patologia acuta.......

Terapia delle comorbidità - non cardiovascolari:

diabete, iperkaliemia, carenza di ferro e cancro. .

Terapia non farmacologica ed educazione/formazione del paziente

e del caregiver.

Gestione post-ricovero dello scompenso cardiaco:

l'ambulatorio divisionale e la rete territoriale 


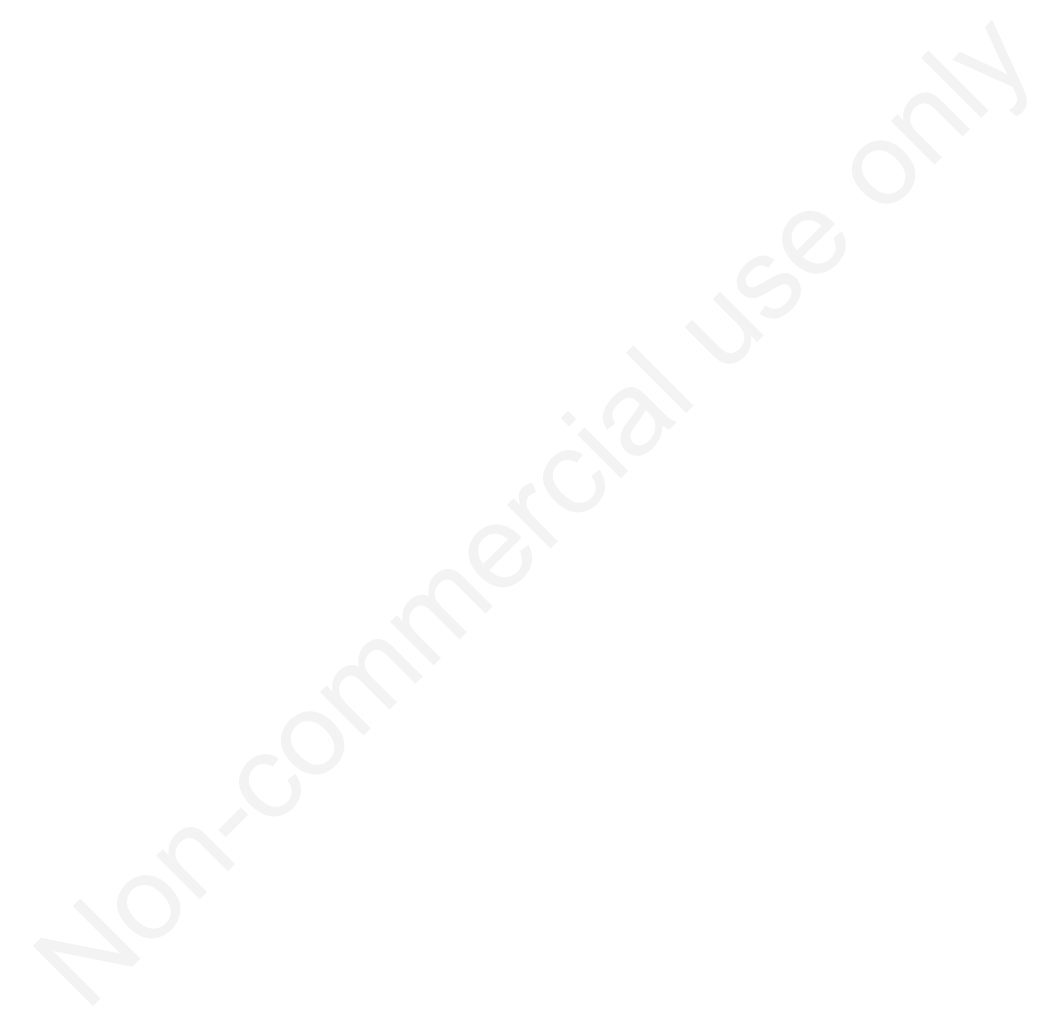




\section{Nuova classificazione dello scompenso cardiaco:}

\section{ridenominazione di Heart failure with mid-range ejection fraction}

\section{in Heart failure with mildly reduced ejection fraction (HFmrEF), con raccomandazioni terapeutiche più precise in questa fascia di pazienti}

Nei pazienti con scompenso cardiaco (SC), la frazione di eiezione ventricolare sinistra (FE) rimane il parametro fondamentale per la diagnosi, la classificazione, la prognosi e le scelte terapeutiche. ${ }^{1}$ Infatti, nonostante alcuni aspetti "discutibili" circa il suo uso clinico, la FE rappresenta il principale indice per la caratterizzazione dello SC ed il criterio primario di inclusione per gli studi clinici sull'insufficienza cardiaca. $^{2}$

Nell'ambito dei tipi di SC, quello a FE ridotta (HFrEF: FE <40\%) è ben caratterizzato ed i risultati di numerosi trials hanno fornito dati per raccomandazioni terapeutiche forti e con elevato livello di evidenza (3).

La definizione di SC a FE preservata $(\mathrm{HF} p \mathrm{EF})$, invece, si riferisce ai pazienti con segni e sintomi di SC ed una FE considerata con differenti valori nel tempo, ma comunque superiori al $40 \%$ ( $>40 \%, \geq 45 \%$ o $>45 \%, \geq 50 \%$ o $>50 \%$ ). Per tale categoria di pazienti, nessuno studio clinico fino ad oggi ha dimostrato benefici evidenti della terapia. ${ }^{3}$

Nelle linee guida ESC HF 2016, è stata introdotta una terza categoria intermedia di SC, definita come $\mathrm{SC}$ con FE di medio "grado" (HFmrEF; con FE compresa tra 40 e $49 \%$ ), che ha incentivato la ricerca sulle caratteristiche nosografiche, la fisiopatologia ed il trattamento dei pazienti con $\mathrm{SC}$ in questo intervallo di $\mathrm{FE}$, meno studiato rispetto a $\mathrm{HF} r \mathrm{EF}$ e $\mathrm{HF} p \mathrm{EF}$, agevolando, inoltre, la diagnosi di SC in un contesto nel quale la $\mathrm{FE}$ non è particolarmente ridotta. ${ }^{4,5} \mathrm{Si}$ è individuato, quindi, un nuovo fenotipo di SC intermedio tra $\mathrm{HF} p \mathrm{EF}$ e $\mathrm{HF} r \mathrm{EF}$, caratterizzato da $\mathrm{FE}$ del $40-49 \%$ associata alla presenza di segni e sintomi di SC, livelli elevati di peptidi natriuretici nel plasma ed evidenza di cardiopatia strutturale (ipertrofia ventricolare sinistra o rimodellamento atriale sinistro) o di disfunzione diastolica. La classe di HFmrEF può includere sia pa-

Corrispondente: Fernando Gallucci.

E-mail: fernando.gallucci62@gmail.com

Articolo pubblicato secondo la Creative Commons Attribution

NonCommercial 4.0 License (CC BY-NC 4.0).

${ }^{\circ}$ Copyright: the Author(s), 2021

Licensee PAGEPress, Italy

QUADERNI - Italian Journal of Medicine 2021; 9(5):e1 zienti la cui FE sia incrementata da $\geq 40 \%$, sia pazienti i cui si sia ridotta da $\geq 50 \% .{ }^{6}$ Esiste una sostanziale sovrapposizione di caratteristiche cliniche, fattori di rischio, tipologia di rimodellamento cardiaco e prognosi fra i tre tipi di SC, anche se diversi studi, pur dimostrando che HFmrEF presenta alcune caratteristiche intermedie tra $\mathrm{HF} r \mathrm{EF}$ e $\mathrm{HF} p \mathrm{EF}$, suggeriscono maggiori somiglianze con $\mathrm{HF} r \mathrm{EF} .^{7}$ I pazienti con $\mathrm{HF} m r \mathrm{EF}$, infatti, sono più frequentemente maschi, sono più giovani ed hanno maggiori probabilità di essere affetti da cardiopatia ischemica $(50-60 \%),{ }^{8,9}$ e minori probabilità di avere fibrillazione atriale e comorbidità non cardiache. Tuttavia, i pazienti ambulatoriali con HF $m r E F$ hanno una mortalità inferiore rispetto a quelli con $\mathrm{HF} r \mathrm{EFe}$ più simile a quelli con $\mathrm{HF} p \mathrm{EF}^{10,11}$

Queste osservazioni giustificano, quindi, l'introduzione del termine SC con FE "moderatamente ridotta" ("heart failure with mildly reduced ejection fraction"), la nuova definizione della classe intermedia di SC che prevede la presenza di sintomi e/o segni di SC, una FE compresa tra 41 e $49 \%$, valori elevati di peptidi natriuretici (BNP $\geq 35 \mathrm{pg} / \mathrm{mL} \quad$ o NTproBNP $\geq 125 \mathrm{pg} / \mathrm{mL}$ ) e altre evidenze di patologia strutturale cardiaca e che ha sostituito la precedente, nelle nuove linee guida ESC 2021 sullo SC.,12 L'esigenza di qesta variazione è stato determinata, da un lato dal riconoscimento che la FE è una variabile a distribuzione continua, la cui misurazione è altamente soggetta alla variabilità intra- e inter-operatore e dall'altro da analisi retrospettive di diversi trial clinici che hanno incluso pazienti con FE tra $40 \%$ e $50 \%$ e che hanno dimostrato come questi pazienti sembrino beneficiare delle stesse terapie che si sono dimostrate efficaci nei pazienti con SC, ribadendo l'ipotesi che tali pazienti siano più simili a quelli con $\mathrm{HF} r \mathrm{EF}$ che a quelli con HF $p E F .{ }^{12,13}$ L'individuazione di questa categoria intermedia di SC ha, quindi, permesso per la prima volta il riconoscimento nelle $\mathrm{LG}$ di un ruolo per beta-bloccanti, ace-inibitori, sartani e sacubitril/valsartan nel trattamento dei pazienti con SC con FE compresa tra 40 e $50 \%$, conferendo una raccomandazione di classe IIb (livello di evidenza C). ${ }^{3}$ Particolarmente convincenti sono state le sottoanalisi dello studio PARAGON-HF, ${ }^{14}$ in cui sacubitril/valsartan ha ridotto l'end-point composito di morte cardiovascolare ed ospedalizzazioni per SC del 22\% nei pazienti con 
$\mathrm{FE}<57 \%$, con un beneficio tanto più marcato quanto più la $\mathrm{FE}$ si riduceva.

\section{Bibliografia}

1. Mele D, Nardozza M, Ferrari R. Left ventricular ejection fraction and heart failure: an indissoluble marriage? Eur J Heart Fail 2018;20:427-430).

2. Lund LH, Vedin O, Savarese G. Is ejection fraction in heart failure a limitation or an opportunity? Eur J Heart Fail 2018 Mar; 20: 431-432.

3. McDonagh TA, Metra M, Adamo M, et al. 2021 ESC Guidelines for the diagnosis and treatment of acute and chronic heart failure. Eur Heart J (2021) 00, 1-128, doi:10.1093/eurheartj/ehab368.

4. Ponikowski P, Voors AA, Anker SD, et al. ESC Scientific Document Group. 2016 ESC Guidelines for the diagnosis and treatment of acute and chronic heart failure: The Task Force for the diagnosis and treatment of acute and chronic heart failure of the European Society of Cardiology (ESC). Developed with the special contribution of the Heart Failure Association (HFA) of the ESC. Eur Heart J 2016;37:2129-2200.

5. Branca L, Sbolli M, Metra M and Fudim M. Heart failure with mid-range ejection fraction: pro and cons of the new classification of Heart Failure by European Society of Cardiology guidelines. ESC Heart Failure 2020; 7: 381-399.

6. Tsuji K, Sakata Y, Nochioka K, et al. CHART-2 Investigators. Characterization of heart failure patients with mid-range left ventricular ejection fraction-a report from the CHART-2 Study. Eur J Heart Fail 2017;19: 12581269.

7. Nauta JF, Hummel YM, van Melle JP, et al. What have we learned about heart failure with mid-range ejection fraction one year after its introduction? Eur J Heart Fail 2017;19:1569-1573).

8. Koh AS, Tay WT, Teng THK, et al. A comprehensive population-based characterization of heart failure with mid-range ejection fraction. Eur J Heart Fail 2017;19: 1624-1634.

9. Vedin O, Lam CSP, Koh AS, et al. Significance of ischemic heart disease in patients with heart failure and preserved, midrange, and reduced ejection fraction: a nationwide cohort study. Circ Heart Fail 2017;10: e003875.

10. Kapoor JR, Kapoor R, Ju C, et al. Precipitating clinical factors, heart failure characterization, and outcomes in patients hospitalized with heart failure with reduced, borderline, and preserved ejection fraction. JACC Heart Fail 2016;4:464-472.

11. Li P, Zhao1 H, Zhang J, Ning Y, et al. Similarities and Differences Between HFmrEF and HFpEF. Front Cardiovasc Med . 2021 Sep 20;8:678614. doi: 10.3389/ fcrm.2021.678614.

12. Lam CSP, Voors AA, Piotr P et al. Time to rename the middle child of heart failure: heart failure with mildly reduced ejection fraction. Eur Heart J (2020) 41, $2353-$ 2355. doi:10.1093/eurheartj/ehaa158).

13. Savarese G, Stolfo D, Sinagra G and Lund LH. Heart failure with mid-range or mildly reduced ejection fraction. Nat Rev Cardiol. 2021 Sep 6 : 1-17. doi: 10.1038/ s41569-021-00605-5.

14. Solomon SD, McMurray JJV, Anand IS, et al. PARAGON-HF Investigators and Committees. Angiotensin-neprilysin inhibition in heart failure with preserved ejection fraction. N Engl J Med 2019;381: 1609-1620. 


\title{
Il nuovo algoritmo per il trattamento dello scompenso cardiaco
}

\section{a frazione di eiezione ridotta:}

\author{
Simultaneous or Rapid Sequence Initiation \\ of Quadruple Medical Therapy for Heart Failure
}

Un'importante novità delle nuove linee guida sullo scompenso cardiaco è rappresentata dal superamento della precedente strategia basata sull'implementazione della terapia step by step in base alla risposta clinica del paziente con l'inserimento delle varie molecole, diuretici, beta-bloccanti, ACE-inibitori ed antialdosteronici. In questa escalation le nuove molecole quali sacubitril/valsartan ed empaglifozin erano relegate in una fase che considereremo dei no responder con persistenza della sintomatologia cardiaca alla terapia tradizionale con ovvio discomfort del paziente nel periodo di upgrade.

La nuova strategia indicata nelle ultime linee guida non solo contempla la possibilità di un'inserimento precoce delle quattro molecole ma ne caldeggia una rapidità di implementazione fino al raggiungimento del target che è quello di ridurre la sintomatologia del paziente nel più breve tempo possibile, che si traduce fortunatamente nella maggior parte dei casi anche con un netto miglioramento dell'emodinamica e della congestione sistemica. Pertanto inizialmente i farmaci venivano inseriti con una latenza abbastanza ampia e con una titolazione graduale, attualmente invece i pazienti potranno essere trattati ab inizio con tutto l'arsenale attualmente disponibile in materia di scompenso cardiaco.

Avremo quindi la possibilità di sartorializzare le terapie sulle caratteristiche dei nostri pazienti senza dover rinunciare ad alcuna molecola e poter scegliere quelle che meglio si performano al fenotipo di paziente con scompenso cardiaco che ci troviamo davanti.

Questo cambio di "rotta" si è reso necessario in considerazione del fatto che nella nostra pratica clinica l' approccio step by step ha dato la possibilità di avere delle terapie veramente ottimizzate e a target in meno del $30 \%$ dei pazienti che arrivano nei nostri reparti ed

Corrispondente: Francesco Dentali.

E-mail: francesco.dentali@asst-settelaghi.it

Articolo pubblicato secondo la Creative Commons Attribution

NonCommercial 4.0 License (CC BY-NC 4.0).

${ }^{\circ}$ Copyright: the Author(s), 2021

Licensee PAGEPress, Italy

QUADERNI - Italian Journal of Medicine 2021; 9(5):e2 ambulatori, ma anche perchè l'associazione delle principali molecole può ridurre la mortalità fino al $73 \%$. L'introduzione delle 4 molecole disease modifying non ha però una precisa indicazione sulle modalità all'interno delle linee guida. Questo probabilmente perchè, nei pazienti con scompenso cardiaco a frazione di eiezione ridotta, l'inserimento dei farmaci non è mai facile nell'immediato in quanto ha comunque una necessità seppur breve di monitoraggio nel tempo per valutare quelle che sono le alterazioni emodinamiche ed elettrolitiche che più spesso vengono a manifestarsi in seguito all'inserimento di una o più delle sopra citate molecole. Talora queste tendono anche a potenziarsi a vicenda impedendo in alcuni casi la possibilità di arrivare a target con una o tutte le terapie in tempi rapidi.

E' vero comunque che parliamo nel caso dello scompenso cardiaco a ridotta frazione di eiezione di pazienti con prognosi severe che vengono spesso ospedalizzati e che tendono a recidivare già nei primi periodi post dimissione con ulteriore aggravio di reingressi e che infine possono aumentare quello che l'outcome di mortalità talora per complicanze cardiovascolari e talora per altre complicanze in corso di degenza.

E'noto come soprattutto negli stadi avanzati dello scompenso cardiaco ed, in particolare nei pazienti con frazione di eiezione ridotta, il rischio indotto dalla congestione sistemica favorisce la mortalità di tipo aritmico e per complicanze non cardiache, rispetto a quella trombotica che è più rappresentata nelle fasi iniziali. Per tale motivo il raggiungimento in tempi brevi di una terapia ottimale per evitare il peggioramento verso le ultime due classi NYHA permette una riduzione di questi eventi.

Inoltre si potrebbe vedere questo cambio di paradigma come uno stimolo per coloro che si occupano di gestione dei pazienti con scompenso cardiaco di sviluppare dei modelli di implementazione terapeutica personalizzati che possano consentire nel maggior numero di pazienti possibili l'utilizzo contemporaneo di tutti e quattro i pilastri farmacologici dello scompenso cardiaco. 


\section{Ottimizzazione della terapia: I'ospedalizzazione come opportunità. Impiego precoce di sacubitril/valsartan nel paziente ospedalizzato, stabilizzato. \\ Effetti sulla riduzione della mortalità e delle re-ospedalizzazioni. Effetti sul rimodellamento cardiaco. Effetti sulla QoL}

L'ottimizzazione della terapia, cioè il "migliore" impiego dei farmaci che i trials randomizzati e controllati hanno dimostrato essere efficaci sugli hard end-points (in particolare mortalità ed ospedalizzazioni), secondo le raccomandazioni delle linee-guida, deve rappresentare un obiettivo di fondamentale importanza da raggiungere. ${ }^{1,2}$

L'ospedalizzazione, pur rappresentando un momento critico nella storia naturale del paziente con scompenso cardiaco (SC), può, o meglio, deve diventare un'opportunità terapeutica, grazie alla possibilità di gestire la transizione dalla terapia della fase acuta al trattamento basato sulle evidenze, possibilmente con l'utilizzo di nuovi presidi terapeutici disponibili. Consente, inoltre, di operare la riconciliazione della terapia farmacologica nelle transizioni di cura, la cui mancata attuazione può causare gravi danni al paziente. ${ }^{3}$

Diverse sono le evidenze in letteratura circa $\mathrm{i}$ benefici dell'inizio durante la degenza ospedaliera di terapie secondo le Linee Guida per lo SC (ad es. con antagonisti recettoriale dei mineralcorticoidi, beta-bloccanti), con dimostrazione che tale approccio comporta un migliore utilizzo dei farmaci e maggiore aderenza e persistenza terapeutica, rafforzandone il razionale. ${ }^{4-6}$

Anche per il sacubitril/valsartan vi sono dati relativi ai vantaggi derivanti dal suo uso precoce, durante la degenza ospedaliera, mostrando di essere in grado di offrire ai pazienti con SC a FE ridotta una protezione anticipata che copre anche la "fase vulnerabile". 7-9

La decisione di iniziare precocemente il trattamento con una bassa dose di un nuovo inibitore neuro-ormonale $(\mathrm{ARNI})$ si è dimostrata superiore rispetto a quella di ritardarlo fino a quando non fosse prima titolato a dose target un altro inibitore già prescritto. Tale strategia, infatti, comporta benefici che non possono essere

Corrispondente: Fernando Gallucci.

E-mail: fernando.gallucci62@gmail.com

Articolo pubblicato secondo la Creative Commons Attribution NonCommercial 4.0 License (CC BY-NC 4.0).

${ }^{\circ}$ Copyright: the Author(s), 2021

Licensee PAGEPress, Italy

QUADERNI - Italian Journal of Medicine 2021; 9(5):e3 ottenuti attendendo prima la titolazione a dose target, ad esempio, di un ACE-inibitore. ${ }^{10}$

Dopo i risultati dello studio PARADIGM-HF, ${ }^{11}$ con significativa riduzione della mortalità e delle riospedalizzazioni in pazienti ambulatoriali con SC a FE ridotta trattati con sacubitril/valsartan rispetto ad enalapril (E), sono stati pubblicati i risultati di altri studi che hanno fornito dati circa l'analoga efficacia e sicurezza dell' $i$ nizio di tale terapia anche in: 1) pazienti recentemente ospedalizzati per episodio di SC acuto; 2) pazienti con diagnosi di SC de novo; 3) pazienti con $\mathrm{SC}$ a FE ridotta non precedentemente trattati con ACEi/ARB.

Lo studio TRANSITION ha evidenziato la sicurezza e la tollerabilità di un inizio precoce di sacubitril/valsartan in pazienti con SC a FE ridotta in pazienti ospedalizzati stabilizzati dopo un episodio acuto con SC pre-esistente o di nuova diagnosi (de novo) con qualsiasi dose di ACEI/ARB utilizzata prima del ricovero, o ACEI/ARB naïve. Gli endpoints primario (numero di pazienti che raggiungevano la dose target di 97/103 mg bid alla decima settimana) e secondari sono stati raggiunti in proporzione simile nei due gruppi di pazienti (inizio terapia pre- o post-dimissione). Anche l'incidenza di eventi avversi seri (AEs) e di sospensione di sacubitril/valsartan per AEs era simile in entrambi i gruppi. Quindi, la dimostrazione che l'inizio precoce in ospedale della terapia con sacubitril/valsartan in un ampio spettro di pazienti stabilizzati dopo un evento di SC acuto è possibile e complessivamente ben tollerato, offre la possibilità di trattare il paziente già durante la fase di ricovero ospedaliero, con la conseguenza di aumentarne l'aderenza alla terapia, monitorarne la tollerabilità ed anticipare gli effetti benefici della terapia. ${ }^{12}$

Lo studio PIONEER-HF che ha confrontato sacubitril/valsartan ed $\mathrm{E}$ in pazienti ricoverati per un episodio di riacutizzazione dello $\mathrm{SC}$ e con elevati livelli di NT-ProBNP, ha messo in evidenza come l'inizio della terapia con sacubitril/valsartan, dopo la stabilizzazione emodinamica, determini un decremento significativo dei livelli di NT-proBNP a 8 settimane rispetto ad E (end-point primario) e come questo effetto sia evidente già nella prima settimana. Le conclusioni dello studio sono state che la terapia con sacubitril/valsartan iniziata durante l'ospedalizzazione (dopo la fase di stabilizzazione emodinamica) è efficace, caratterizzata da un pro- 
filo di sicurezza favorevole, comporta benefici che si mantengono nella fase di transizione domiciliare postricovero e per tutto il «periodo vulnerabile». ${ }^{13}$

La riduzione dei livelli di NT-proBNP in pazienti con SC (end-point surrogato), non rappresenta un mero dato di miglioramento laboratoristico ma, dal punto di vista clinico, si associa ad un minor rischio di esiti negativi come morte cardiovascolare o ospedalizzazione per SC.

Il risultato è sostenuto dalla metanalisi di Savarese et al..$^{14}$ che ha evidenziato come la riduzione dei livelli di peptidi natriuretici indotta dal trattamento avesse ridotto significativamente il rischio di ospedalizzazione per peggioramento dello SC.

Si può affermare, quindi, che sacubitril/valsartan può essere somministrato anche in regime di ricovero ospedaliero in pazienti stabilizzati emodinamicamente, per ridurre l'attivazione neuro-ormonale e diminuire il rischio di successiva ospedalizzazione per SC. ${ }^{15}$

Abbiamo, poi, dimostrazione (Studio PROVE-HF) che il decremento dei valori di NT-proBNP risulta strettamente correlato anche al miglioramento dei parametri di rimodellamento cardiaco, come FEVS, LVEDVi/LVESVi, LAVi e funzione diastolica misurata come rapporto E/e' ("rimodellamento inverso") con miglioramento della prognosi. ${ }^{16}$

Concordi con questi dati sono le conclusioni dello studio EVALUATE-HF, in cui il sacubitril/valsartan ha migliorato significativamente i principali parametri morfo-funzionali cardiaci. ${ }^{17}$

Quindi, sacubitril/valsartan è in grado di contrastare il "deletereo" rimodellamento cardiaco caratteristico dello $\mathrm{SC}$ a FE ridotta, determinando un'azione favorevole (rimodellamento inverso) che può spiegare meccanicisticamente gli effetti clinici benefici della terapia, il cui marcatore (NT-proBNP) è di facile interpretazione, non solo da parte dello specialista.

Infine, $\mathrm{i}$ dati derivati dallo studio PARADIGM-HF dimostrano che gli score di sintesi clinica KCCQ ed i punteggi di riepilogo generale KCCQ erano migliori nei pazienti con SC a FE ridotta trattati con sacubitril/valsartan rispetto a quelli trattati con $\mathrm{E}$, con persistenza del vantaggio durante il follow-up oltre gli 8 mesi e fino ai 36 mesi, realizzando una migliore performance sulla HRQL. ${ }^{18}$

\section{Bibliografia}

1. Ameri P, Amico AF, Battagliese A, et al. L'ottimizzazione terapeutica nel percorso del paziente con scompenso cardiaco. G Ital Cardiol 2020;21(12Suppl.1): e3-e15|DOI 10.1714/3479.34615.

2. McDonagh TA, Metra M, Adamo M, et al. 2021 ESC Guidelines for the diagnosis and treatment of acute and chronic heart failure. ESC Scientific Document Group. Eur Heart J. 2021 Sep 21;42(36):3599-3726. doi: 10.1093/eurheartj/ehab368.

3. Di Lenarda A, Colivicchi F, Gabrielli D. L'ospedalizzazione per scompenso cardiaco acuto: come un evento critico può diventare un'opportunità clinica. G Ital Cardiol 2019;20:690-3.

4. Maggioni AP. Epidemiology of Heart Failure in Europe.
Heart Fail Clin. 2015 Oct;11(4):625-35. doi: 10.1016/j .hfc.2015.07.015. Epub 2015 Aug 8.

5. Atherton JJ, Hayward CS, Wan Ahmad WA, et al. Patient characteristics from a regional multicenter database of acute decompensated heart failure in Asia Pacific (ADHERE International-Asia Pacific). J Card Fail. 2012 Jan;18(1):82-8. doi: 10.1016/j.cardfail.2011.09.003. Epub 2011 Oct 8.

6. Gattis WA, O'Connor CM, Gallup DS, et al., IMPACTHF Investigators and Coordinators. Predischarge initiation of carvedilol in patients hospitalized for decompensated heart failure: results of the Initiation Management Predischarge: Process for Assessment of Carvedilol Therapy in Heart Failure (IMPACT-HF) trial. J Am Coll Cardiol. 2004 May 5;43(9):1534-41. doi: 10.1016/j.jacc.2003.12.040.

7. Packer M, McMurray JJV, Desai AS et al. PIONEERHF. Angiotensin receptor neprilysin inhibition compared with enalapril on the risk of clinical progression in surviving patients with heart failure. Circulation 2015;131 (1):54-61.

8. Desai AS, McMurray JJV, Packer M et al. Effect of the angiotensin-receptor-neprilysin inhibitor LCZ696 compared with enalapril on mode of death in heart failure patientsEur Heart J 2015;36(30):1990-7.

9. Volpe M. Natriuretic peptides and cardio-renal disease. Int J Cardiol. 2014 Oct 20;176(3):630-9. doi: 10.1016/j.ijcard.2014.08.032. Epub 2014 Aug 12.

10. Packer M. Love of Angiotensin-Converting Enzyme Inhibitors in the Time of Cholera. JACC Heart Fail. 2016 Apr 12;S2213-1779(16)30045-2. doi: 10.1016/j. jchf.2016.02.012.

11. McMurray JJ, Packer M, Desai AS, et al.; PARADIGMHF Investigators and Committees. Angiotensinneprilysin inhibition versus enalapril in heart failure. $\mathrm{N}$ Engl J Med 2014;371:993-1004.

12. Wachter R, Senni M, Belohlavek J, et al. TRANSITION Investigators. Initiation of sacubitril/valsartan in haemodynamically stabilised heart failure patients in hospital or early after discharge: primary results of the randomised TRANSITION study. Eur J Heart Fail 2019;21:998-1007.

13. Velazquez EJ, Morrow DA, DeVore AD, et al.; PIONEER-HF Investigators. Angiotensin-neprilysin inhibition in acute decompensated heart failure. N Engl J Med 2019;380:539-48.

14. Savarese G, Musella F, D'Amore C, et al. Changes of natriuretic peptides predict hospital admissions in patients with chronic heart failure: a meta-analysis. JACC Heart Fail 2014;2:148-58.

15. Seferovic PM, Ponikowski P, Anker SD et al. Eur J Heart Fail. 2019 May 26. Clinical practice update on heart 2019: pharmacotherapy, procedures, devices and patient management- An expert consensus meeting report of The Heart Failure Association of the European Society of Cardiology.

16. Januzzi JL, Butler J, Fombu E, et al. Rationale and methods of the Prospective Study of Biomarkers, Symptom Improvement, and Ventricular Remodeling During sacubitril/valsartan Therapy for Heart Failure (PROVEHF). Am Heart J 2018;199:130-6.

17. Desai AS, Solomon SD, Shah AM, et al. EVALUATEHF Investigators. Effect of sacubitril-valsartan vs enalapril on aortic stiffness in patients with heart failure and reduced ejection fraction: a randomized clinical trial. JAMA 2019;322:1-10.

18. Lewis EF, Claggett BL, McMurray JJ, et al. Health-related quality of life outcomes in PARADIGM-HF. Circ Heart Fail 2017;10(8):e003430. 


\section{Sicurezza dell'uso di sacubitril/valsartan anche in presenza di comorbidità e gestione dell'ipotensione}

Dopo la dimostrazione dell'efficacia di sacubitril/valsartan con lo studio registrativo, diverse analisi post-hoc del PARADIGM hanno confermato l'efficacia e dimostrato la sicurezza del farmaco anche riguardo alle comorbidità spesso compresenti allo scompenso cardiaco, in particolare nei pazienti polipatologici affetti da insufficienza renale cronica, diabete mellito, ipertensione arteriosa e fibrillazione atriale.

Nel 2017 Seferovic et al. hanno dimostrato che, prospetticamente, sacubitril/valsartan riduce significativamente l'emoglobina glicata nei pazienti diabetici rispetto all'enalapril (a 3 anni: - 0.14, 0.06- 0.23, p: 0.0055). ${ }^{1}$

I pazienti in trattamento con sacubitril/valsartan, rispetto ai pazienti in trattamento con enalapril, vanno incontro a necessità di antidiabetico orale in percentuale minore (HR 0.77, 95\% CI 0.58-1.02; $\mathrm{p}=0.07$ ) e a minore necessità di iniziare la terapia insulinica $(\mathrm{HR}=0.71(0.56$ $0.90) ; p=0.005)$ a fronte di un rischio di ipoglicemie sovrapponibile (HR 1.18, 95\% CI 0.79-1.76; $\mathrm{p}=0.42$ ).

L'analisi post-hoc dell'outcome renale composito (malattia renale allo stadio terminale o riduzione dell'eGFR $\geq 50 \%$ ) ha dimostrato che questo si verificava con minore frequenza nel gruppo sacubitril/valsartan (HR 0,63; IC 95\% 0,42-0,95; $\mathrm{p}=0,028$ ) rispetto a enalapril e in entrambi i sottogruppi con e senza malattia renale cronica. ${ }^{2}$

Questa differenza significativa risultava indipendente dalla pressione sistolica basale, dalla creatinina sierica, dalla storia anamnestica di ipertensione arteriosa, scompenso cardiaco o coronaropatia.

In associazione all'antialdosteronico, sacubitril/valsartan si è dimostrato più sicuro dell'enalapril anche circa il rischio di iperkaliemia severa o moderata. ${ }^{3}$

Anche l'iperuricemia è una condizione frequentemente associata a patologie cardiovascolari ed in particolare allo scompenso cardiaco acuto e cronico.

L'iperuricemia è da sempre associata ad outcomes cardiovascolari sfavorevoli ma non era chiaro se rappresentasse un fattore prognostico sfavorevole indipen-

Corrispondente: Maurizio Cavalleri.

E-mail: mcavalleri80@gmail.com

Articolo pubblicato secondo la Creative Commons Attribution

NonCommercial 4.0 License (CC BY-NC 4.0).

${ }^{\circ}$ Copyright: the Author(s), 2021

Licensee PAGEPress, Italy

QUADERNI - Italian Journal of Medicine 2021; 9(5):e4 dente, poiché è una condizione che si associa spesso ad altri fattori confondenti quali l'uso di diuretici e una funzione renale alterata, che di per se rappresentano già fattori prognosticamente sfavorevoli. Ancora una volta un'analisi post-hoc dello stuio PARADIGM ha fornito importanti informazioni al riguardo, dimostrando che l'iperuricemia è effettivamente un fattore prognostico indipendente per l'outcome primario di mortalità cardiovascolare, ospedalizzazione per scompenso cardiaco e mortalità per tutte le cause; lo studio conferma peraltro la sicurezza del sacubitril/valsartan nel determinare una riduzione significativa dell'iperuricemia rispetto all'enalapril. ${ }^{4}$

I benefici di sacubitril/valsartan rispetto all'enalapril sono indipendenti dai valori basali di pressione arteriosa sistemica: i pazienti con valori moderatamente inferiori (PS $<$ di $110 \mathrm{mmHg}$ ) hanno avuto un beneficio netto superiore rispetto ai pazienti con valori pressori più alti, pur a scapito di effetti collaterali di ipotensione, senza risvolti rilevanti sulla prognosi. E' quindi opportuno non procrastinare l'inizio del farmaco solo per il timore degli effetti collaterali da ipotensione, ma piuttosto, mettere in atto ogni strategia atta a ridurre il rischio di eventuali eventi ipotensivi (ad esempio modulare la terapia diuretica, sostituire il betabloccante quale atenololo con bisoprololo, correggere l'ipovolemia, sospendere altri farmaci ipotensivi,...). ${ }^{5}$

In questo senso l'ospedalizzazione deve essere vista come un'opportunità, al fine di inquadrare il paziente nella sua complessità e per iniziare e titolare nuovi farmaci come sacubitril/valsartan, secondo le indicazioni che vengono fornite dalle Linee-guida.

Questo importante e innovativo concetto è avvalorato dai risultati dello studio TRANSITION. ${ }^{6}$ Questo studio infatti ha confrontato due ipotesi: iniziare sacubitril/valsartan già durante la degenza ospedaliera, prima della dimissione $v s$ inziare la terapia post-dimissione. Lo studio ha dimostrato che non vi sono state differenze significative in termini di tollerabilità tra le due procedure, con percentuali analoghe di pazienti che raggiungevano la dose target di farmaco a 10 settimane di follow-up e con un profilo di safety sovrapponibile in entrambi i gruppi. Questo significa quindi che iniziare sacubitril/valsartan precocemente, già durante il ricovero, a stabilizzazione raggiunta di uno scompenso de novo o riacutizzato, è una strategia efficace e sicura che permette di ottenere una maggiore protezione della fase vulnerabile dei 30 giorni post-ricovero. 


\section{Bibliografia}

1. Seferovic JP, Claggett B, Seidelmann SB, Seely EW, Packer M, Zile MR, Rouleau JL, Swedberg K, Lefkowitz M, Shi VC, Desai AS, McMurray JJV, Solomon SD. Effect of sacubitril/valsartan versus enalapril on glycaemic control in patients with heart failure and diabetes: a posthoc analysis from the PARADIGM-HF trial. Lancet Diabetes Endocrinol. 2017 May;5(5):333-340. doi: 10.1016/ S2213-8587(17)30087-6. Epub 2017 Mar 18. PMID: 28330649; PMCID: PMC5534167.

2. Damman K, Gori M, Claggett B, Jhund PS, Senni M, Lefkowitz MP, Prescott MF, Shi VC, Rouleau JL, Swedberg K, Zile MR, Packer M, Desai AS, Solomon SD, McMurray JJV. Renal Effects and Associated Outcomes During Angiotensin-Neprilysin Inhibition in Heart Failure. JACC Heart Fail. 2018 Jun;6(6):489-498. doi: 10.1016/j.jchf.2018.02.004. Epub 2018 Apr 11. PMID: 29655829.

3. Desai AS, Vardeny O, Claggett B, et al. Reduced Risk of Hyperkalemia During Treatment of Heart Failure With Mineralocorticoid Receptor Antagonists by Use of sacubitril/valsartan Compared With Enalapril: A Secondary Analysis of the PARADIGM-HF Trial. JAMA Cardiol. 2017;2(1):79-85. doi:10.1001/jamacardio.2016.4733.

4. Mogensen UM, Køber L, Jhund PS, Desai AS, Senni M, Kristensen SL, Dukát A, Chen CH, Ramires F, Lefkowitz MP, Prescott MF, Shi VC, Rouleau JL, Solomon SD,
Swedberg K, Packer M, McMurray JJV; PARADIGMHF Investigators and Committees. sacubitril/valsartan reduces serum uric acid concentration, an independent predictor of adverse outcomes in PARADIGM-HF. Eur J Heart Fail. 2018 Mar;20(3):514-522. doi: 10.1002/ejhf. 1056. Epub 2017 Nov 30. PMID: 29193563; PMCID: PMC6607477.

5. Böhm M, Young R, Jhund PS, Solomon SD, Gong J, Lefkowitz MP, Rizkala AR, Rouleau JL, Shi VC, Swedberg K, Zile MR, Packer M, McMurray JJV. Systolic blood pressure, cardiovascular outcomes and efficacy and safety of sacubitril/valsartan (LCZ696) in patients with chronic heart failure and reduced ejection fraction: results from PARADIGM-HF. Eur Heart J. 2017 Apr 14;38(15):1132-1143. doi: 10.1093/eurheartj/ehw570. PMID: 28158398; PMCID: PMC6251522.

6. Wachter R, Senni M, Belohlavek J, Straburzynska-Migaj E, Witte KK, Kobalava Z, Fonseca C, Goncalvesova E, Cavusoglu Y, Fernandez A, Chaaban S, Bøhmer E, Pouleur AC, Mueller C, Tribouilloy C, Lonn E, A L Buraiki J, Gniot J, Mozheiko $M$, Lelonek $M$, Noè $A$, Schwende H, Bao W, Butylin D, Pascual-Figal D; TRANSITION Investigators. Initiation of sacubitril/valsartan in haemodynamically stabilised heart failure patients in hospital or early after discharge: primary results of the randomised TRANSITION study. Eur J Heart Fail. 2019 Aug;21(8): 998-1007. doi: 10.1002/ejhf.1498. Epub 2019 May 27. PMID: 31134724. 


\section{Intervento di ottimizzazione della terapia anche nel paziente con scompenso cardiaco cronico ricoverato per altra patologia acuta}

La degenza ospedaliera, oltre a rappresentare il momento in cui si trattano le riacutizzazioni delle principali patologie croniche note, prima tra le quali lo scompenso cardiaco cronico, è anche il momento cruciale nella gestione di questa patologia e nell'ottimizzazione della terapia.

Se è vero che la ricognizione farmacologica all'ingresso ci permette di capire quale sia il background generale del paziente e le diverse modalità di gestione delle proprie patologie, l'inserimento di nuove terapie è auspicabile venga fatto precocemente, qualora ci siano le condizioni, in un regime dove è possibile uno stretto monitoraggio qual è quello dei reparti di degenza.

Questo perchè l'implementazione di nuove terapie in pazienti cronici e talora con plurime comorbidità non è scevra da problematiche di tollerabilità, che sono causa di scarsa aderenza terapeutica nel post-dimissione. Tra queste quelle con sacubritril/valsartan e empaglifozin erano in passato appannaggio di un regime post-dimissione ambulatoriale dove era decisamente più difficile valutare in maniera completa le fluttuazioni cliniche e parametriche nel primo inserimento di una nuova molecola. Se poi come in questo caso vi sono associate delle modificazioni emodinamiche talora potenziate dall'interazione con le restanti terapie già in atto, diventa evidente come il paziente possa essere indotto ad una modifica autonoma della terapia, o come talora il curante, sia esso specialista o medico di medicina generale possa modificare la stessa in base a quanto riferito dal paziente per impossibilità, come è avvenuto nell'ultimo periodo causa pandemia, di valutare personalmente le condizioni dell'assistito.

Avendo invece la possibilità di inserire la terapia direttamente durante il periodo di degenza una volta raggiunta la stabilità emodinamica dopo l'evento acuto, ci troviamo nella condizione di monitorare quella che è la tolleranza al nuovo farmaco valutando l'andamento dei vari parametri vitali (pressione arteriosa, frequenza cardiaca e diuresi), delle modificazioni plasmatiche degli elettroliti e cataboliti (creatinina, sodio, potassio e calcio) e della sintomatologia del paziente. Allo stesso tempo in questo modo è possibile educare il paziente all'assunzione del farmaco e alle eventuali fisiologiche fluttuazioni di alcuni parametri vitali, che comunque rientrano nei range di normalità, in modo da non costituire una causa di allarme e focalizzarsi invece su quei segni e sintomi che nel caso dovranno indirizzarlo verso una rivalutazione clinica a breve.

Questo nuovo approccio consente di migliorare l'aderenza alla terapia grazie anche alla buona tollerabilità dell'assunzione nel breve tempo post acuzie. Il tutto si traduce in un intervento precoce sull'attivazione neuro-ormonale con rapida riduzione del NT-proBNP e quindi la possibilità di incidere in maniera migliorativa sulle percentuali di mortalità e reospedalizzazione.

Questo ovviamente renderà necessario l'inserimento nei prontuari ospedalieri delle varie aziende sanitarie questi nuovi farmaci con eventuali facilitazioni nella prescrizione al domicilio almeno del primo periodo di terapia fino alla rivalutazione ambulatoriale.

Altro vantaggio è quello che comunque nel breve periodo sarà necessario creare un percorso di followup anche di breve durata per la conferma dell'aderenza terapeutica e per eventuale titolazione della terapia in base alla risposta clinica ed alla tolleranza (percorso di più breve durata vista l'indicazione a rapido upgrade).
Corrispondente: Francesco Dentali.

E-mail: francesco.dentali@asst-settelaghi.it

Articolo pubblicato secondo la Creative Commons Attribution NonCommercial 4.0 License (CC BY-NC 4.0).

${ }^{\circ}$ Copyright: the Author(s), 2021

Licensee PAGEPress, Italy

QUADERNI - Italian Journal of Medicine 2021; 9(5):e5 


\section{Terapia delle comorbidità - non cardiovascolari: diabete, iperkaliemia, carenza di ferro e cancro}

Il recente aggiornamento delle Linee Guida (LG) ESC esprime raccomandazioni forti per il trattamento di alcune delle principali condizioni associate allo scompenso cardiaco (SC) ${ }^{1}$.

L'insufficienza renale cronica è un fattore prognostico indipendente di aumentata mortalità e morbidità nello scompenso cardiaco. In taluni casi alterazioni della creatinina sierica non corrispondono ad un outcome peggiore per se: ad esempio quando viene iniziata la terapia con sacubitril/valsartan, l'iniziale riduzione della pressione di filtrazione glomerulare, può ridurre il GFR e aumentare la creatinina. Tuttavia si tratta nella maggior parte dei casi di una fluttuazione transitoria, reversibile, che si traduce, al contrario, in una maggiore protezione del filtrato renale e degli outcome clinici del paziente a lungo termine.

Si è già accennato all' analisi post-hoc del PARADIGM che ha dimostrato la sicurezza di sacubitril/valsartan nel paziente diabetico. A lungo termine sacubitril/valsartan permette un miglior controllo dell' $\mathrm{HbA} 1 \mathrm{c}$ rispetto agli ACE-I e un minor ricorso all'insulina. Di particolare interesse saranno i dati di real-world sull'utilizzo già in prima linea di sacubitril/valsartan nel paziente scompensato e diabetico, eventualmente in associazione ad una glifozina (categoria di antidiabetici di nuovo utilizzo nel paziente con diabete mellito e SC) in grado di portare benefici nell'ambito del diabete, ma anche sugli outcome cardiovascolari dello scompensato di per se.

Dallo studio PROVE -HF sappiamo che la riduzione precoce dell'NT-proBNP da parte di sacubitril/valsartan si traduce in un rimodellamento inverso cardiaco e un miglioramento di tutti i principali parametri funzionali ecocardiografici. ${ }^{2}$ Una recente sotto-analisi dello studio ha dimostrato che questo beneficio funzionale e clinico è confermato anche nel sottogruppo dei pazienti diabetici e questo è un dato rilevante considerando che i pazienti con scompenso

Corrispondente: Maurizio Cavalleri.

E-mail: mcavalleri80@gmail.com

Articolo pubblicato secondo la Creative Commons Attribution

NonCommercial 4.0 License (CC BY-NC 4.0).

${ }^{\circ}$ Copyright: the Author(s), 2021

Licensee PAGEPress, Italy

QUADERNI - Italian Journal of Medicine 2021; 9(5):e6 cardiaco e diabete hanno una prognosi peggiore rispetto ai pazienti non diabetici. ${ }^{3}$

La sideropenia e l'anemia sono condizioni che tipicamente associate allo SC e rappresentano fattori di rischio per una maggiore mortalità cardiovascolare e per tutte le cause. La sideropenia, a prescindere dall'anemia, è presente fin nell' $80 \%$ dei casi di scompenso cardiaco acuto ed ha una genesi multifattoriale ancora in parte sconosciuta. Di fatto, però, la sideropenia peggiora la prognosi dello scompenso cardiaco stesso riducendo la capacità funzionale, la funzione muscolare e contribuendo alla fragilità del paziente. La LG raccomanda quindi uno screening regolare per anemia e sideropenia nei Pazienti con scompenso monitorando l'emocromo, la ferritina sierica e TSAT. Viene indicato il supplemento marziale ev con ferrocarbossimaltosio nei pazienti sintomatici con $\mathrm{SC}$ a FE ridotta a meno del $45 \%$, e ferritina meno di $100 \mathrm{ng} / \mathrm{mg}$ o $100-199$ ng/Ml e TSAT meno del $20 \%$, allo scopo di ridurre $\mathrm{i}$ sintomi e migliorare la qualità di vita.

La disionia, e in particolare l'iperkaliemia, si associa frequentemente allo scompenso cardiaco, prevalentemente su base iatrogena. $\mathrm{Si}$ associa frequentemente all'uso dei RAAS-I e all'insufficienza renale. In acuto nell'iperkaliemia severa, la terapia è quella tradizionale con calcio ev o sodio bicarbonato, glucosata con insulina e beta agonisti.

L'iperkaliemia è il principale fattore condizionante l'uso degli ACE-I e degli antialdosteronici, anche se, come detto in precedenza, dalle sottoanalisi del PARADIGM sappiamo che con sac/val l'incidenza di questa complicanza è inferiore rispetto a quella riscontrata con l'uso degli ACE-I.

Sia in acuto che in cronico è indicato l'uso dei chelanti del potassio, che ne riducono l'assorbimento intestinale. In particolare sono stati recentemente introdotti due nuovi farmaci, il Patiromer e il sodio zirconio glicosilato, che aumentano l'escrezione fecale di potassio. Entrambi sono efficaci nel normalizzare i livelli di potassio, mantenendo una normale kaliema nel tempo e riducendo le recidive. L'uso di questi prodotti contestualmente agli ACE-I e agli antialdosteronici riduce il rischio di iperkaliermia e permette di titolare i farmaci cardioattivi mantenendone il beneficio clinico.

Anche il cancro può spesso associarsi a SC come sommatoria di più fattori, i chemioterapici, la malattia neoplastica stessa e i fattori di rischio cardiovascolari preesistenti. 
Per questo motivo tutti i pazienti con diagnosi di cancro che devono intraprendere una terapia attiva citoriduttiva dovrebbero essere sottoposti regolarmente ad una valutazione del rischio cardiovascolare prima , durante e dopo la terapia. La LG suggerisce di utilizzare una stratificazione del rischio $\mathrm{CV}$ come la HFA-ICOS risk assessment. La stratificazione di rischio prevede una valutazione clinica, l'ECG, l'ecocardiografia e i biomarkers cardiaci. I pazienti a rischio intermedio-alto necessiteranno di un monitoraggio più stretto durante la chemioterapia con controlli seriati dei biomarkers e dell'ECG.

Per i pazienti a maggior rischio di cardiotossicità è particolarmente indicata una valutazione da parte di un servizio di cardio-oncologia, ove disponibile.

\section{Bibliografia}

1. Theresa A McDonagh et al. ESC Scientific Document Group, 2021 ESC Guidelines for the diagnosis and treatment of acute and chronic heart failure: Developed by the Task Force for the diagnosis and treatment of acute and chronic heart failure of the European Society of Cardiology (ESC) With the special contribution of the Heart Failure Association (HFA) of the ESC, European Heart Journal, Volume 42, Issue 36, 21 September 2021, Pages 3599-3726, https://doi.org/10.1093/eurheartj/ehab368.

2. Januzzi JL, Prescott MF, Butler J, et al. Association of Change in N-Terminal Pro-B-Type Natriuretic Peptide Following Initiation of Sacubitril-Valsartan Treatment With Cardiac Structure and Function in Patients With Heart Failure With Reduced Ejection Fraction. JAMA. 2019;322(11):1085-1095. doi:10.1001/jama.2019.12821.

3. Khan MS, Felker GM, Piña IL, Camacho A, Bapat D, Ibrahim NE, Maisel AS, Prescott MF, Ward JH, Solomon SD, Januzzi JL, Butler J. Reverse Cardiac Remodeling Following Initiation of sacubitril/valsartan in Patients With Heart Failure With and Without Diabetes. JACC Heart Fail. 2021 Feb;9(2):137-145. doi: 10.1016/j.jchf.2020.09.014. Epub 2020 Dec 9. PMID: 33309581 . 


\section{Terapia non farmacologica ed educazione/formazione del paziente e del caregiver}

La terapia non farmacologica del paziente con scompenso cardiaco ha un ruolo fondamentale, spesso non adeguatamente valorizzato, per la gestione della malattia nel lungo periodo al fine di ridurre le riacutizzazioni di patologia e di aumentare la consapevolezza di malattia, strumenti necessari per una ottimizzazione delle cure. Lo scompenso cardiaco, come molte altre patologie croniche, necessita per un trattamento ottimale, l'affiancamento di terapie farmacologiche e nutrizionali/comportamentali che prevedono l'educazione e la formazione del paziente e del care-giver come individuato anche nelle più recenti linee guida. ${ }^{1}$

Il primo punto di partenza per garantire un'ottimale alleanza terapeutica al fine di ottimizzare i risultati delle cure è quello di rendere consapevoli della malattia paziente, familiari e care-giver. La consapevolezza di malattia è necessaria per far comprendere al paziente ed al suo entourage le possibilità di gestione della patologia cronica nella migliore direzione. Inconsapevolezza, non adeguata comprensione del significato della patologia e delle opzioni e modalità di trattamento, sono fattori che limitano notevolmente i risultati terapeutici del trattamento dello scompenso cardiaco. Le evidenze in merito ad effetti di intervento sulla consapevolezza e sulla capacità di auto(co)gestione nei pazienti con scompenso cardiaco sono molto limitate, tuttavia esistono dati favorevoli che indicano un minore tasso di riospedalizzazioni, mortalità e miglioramento della qualità di vita (Quality Of Life-QOL) valutata con questionari mirati e riproducibili. ${ }^{2}$

Esistono in letteratura numerose modalità di intervento per aumentare la consapevolezza dello scompenso cardiaco cronico e la sua gestione, quali fornire informazioni scritte (brochure), colloqui ripetuti, diari di malattia, condivisione di informazioni fra pazienti ("peer-to-peer" interventions), ma non esistono indicazioni definitive su quale approccio sia migliore. Sicuramente non esiste uno strumento valido per tutti

Corrispondente: Filippo Pieralli.

E-mail: filippopieralli@gmail.com

Articolo pubblicato secondo la Creative Commons Attribution

NonCommercial 4.0 License (CC BY-NC 4.0).

${ }^{\circ}$ Copyright: the Author(s), 2021

Licensee PAGEPress, Italy

QUADERNI - Italian Journal of Medicine 2021; 9(5):e7 (one tool does'nt fit all) poiché il tipo e la modalità di comunicazione e comprensione dipende dalla singola persona e dal suo contesto sociale, economico e culturale. ${ }^{3}$

I campi principali di intervento educazionale non farmacologico sui quali agire e fornire informazioni dettagliate e consapevoli nei pazienti con scompenso cardiaco sono i seguenti:

a. Rendere consapevoli della patologia, delle cause che la determinano, dei sintomi, della prognosi e delle opzioni e scelte terapeutiche.

Ottimizzare l'aderenza ai trattamenti farmacologici semplificando gli schemi di terapia che devono essere chiari ed in forma scritta ben comprensibile. Gli smartphone e app salute possono offrire vantaggi per l'educazione, ricordare gli orari e tracciare l'aderenza ai trattamenti.

b. Informare sugli effetti favorevoli e gli effetti avversi (saperli intercettare e gestire) delle terapie farmacologiche e dei dispositivi impiantabili (es. scariche inappropriate del ICD).

c. Motivare il paziente ad un'attività fisica regolare insegnando a riconoscere le limitazioni funzionali necessarie (attività fisica adattata). Eventualmente avviare a programmi riabilitativi strutturati i pazienti con scompenso cardiaco in forma più grave.

d. Informare sull'importanza del riposo notturno; intercettare e trattare eventuali disturbi del sonno. L'attività sessuale è generalmente sicura e devono essere fornite adeguate informazioni sulla modalità e sulla gestione di eventuali terapie per la disfunzione erettile.

e. Informare adeguatamente sulla necessità di limitare l'introduzione di fluidi e sale. In generale non oltre i 2 litri al giorno, con restrizioni maggiori per i pazienti più gravi e concomitanti segni di congestione e/o iponatriemia. Allo stesso tempo informare sul mantenimento di adeguata idratazione nei periodi più caldi.

f. Educare al mantenimento di un peso stabile, valutato quotidianamente al mattino, e alla gestione di incrementi repentini del peso corporeo ( $>2 \mathrm{~kg}$ in 3 giorni) con incremento della terapia diuretica e/o richiesta di intervento medico.

g. Informare sulla necessità di una sana ed equilibrata alimentazione che privilegi cibi freschi, in particolare frutta, verdura, pesce e carni bianche, ed evitare cibi insaccati, inscatolati e conservati, e ricchi di grassi. Limitare l'uso di alcol all'equivalente di 
1 bicchiere di vino al giorno. Mantenere un peso corporeo nei limiti del normopeso (BMI 20-25) evitando il sovrappeso/obesità, ma anche la malnutrizione ricorrendo eventualmente ad integratori alimentari. Evitare il fumo, indicando anche il riferimento a centri antifumo.

h. Educare ad intercettare sintomi associati della sfera psicologica, quali depressione e ansia, al fine di avviare un trattamento mirato.

i. Informare sulla necessità di eseguire le vaccinazioni indicate per COVID-19, polmonite pneumococcica, virus influenzale, virus herpes zoster (VZV) per proteggersi da complicanze infettive causa di riacutizzazioni e incremento mortalità.

\section{Bibliografia}

1. McDonagh TA, Metra M, Adamo M et al. ESC Guidelines for the diagnosis and treatment of acute and chronic heart failure: Developed by the Task Force for the diagnosis and treatment of acute and chronic heart failure of the European Society of Cardiology (ESC) With the special contribution of the Heart Failure Association (HFA) of the ESC, European Heart Journal, Volume 42, Issue 36, 21 September 2021, Pages 3599-3726, https://doi.org/ 10.1093/eurheartj/ehab36. Maddox TM, Januzzi JL, Allen L, et al. 2021 Update to the 2017 ACC Expert Consensus Decision Pathway for Optimization of Heart Failure Treatment: Answers to 10 Pivotal Issues About Heart Failure With Reduced Ejection Fraction: A Report of the American College of Cardiology Solution Set Oversight Committee. J Am Coll Cardiol. 2021 Feb, 77 (6) 772-810. doi/10.1016/j.jacc.2020.11.022].

2. Jonkman NH, Westland H, Groenwold RH, et al. What are effective program characteristics of self management interventions in patients with heart failure? An individual patient data meta-analysis. J Card Fail 2016;22:861-871.

3. Lainscak M, Blue L, Clark AL, et al. Self-care management of heart failure: practical recommendations from the Patient Care Committee of the Heart Failure Association of the European Society of Cardiology. Eur J Heart Fail 2011;13:115-126. Patient experience in adult NHS services: improving the experience of care for people using adult NHS services Clinical guideline Published: 24 February 2012 www.nice.org.uk/guidance/cg138. 


\section{Gestione post-ricovero dello scompenso cardiaco: l'ambulatorio divisionale e la rete territoriale}

Il Piano Nazionale della Cronicità in Italia, che si propone di delineare un Sistema Nazionale omogeneo, volto a prevenire e gestire la cronicità, è stato recepito dalle regioni in modo piuttosto difforme anche se in tutto il territorio nazionale sono stati istituiti gli ambulatori dedicati alla gestione dello scompenso cardiaco.

L'organizzazione deve prevedere la creazione di una rete strutturata che raccolga strutture, dotazioni e competenze di livello crescente alle quali riferirsi in collegamento funzionale.

Gli ambulatori per lo scompenso cardiaco (SC) possono essere classificati come:

- Ambulatorio territoriale per SC.

- Ambulatorio, Centro Ospedaliero per SC.

- Ambulatorio/unità per SC avanzato e terapia sostitutiva.

Gli ambulatori e i presidi territoriali lavorano sul territorio, nell'ambito di forme organizzative complesse della Medicina Generale:

- Unità Territoriale di assistenza primaria (UCCP).

- Case della Salute.

- Nell'ambito della Specialistica Ambulatoriale.

- In Strutture Intermedie di Lungodegenza.

- In Strutture Riabilitative Cardiologiche.

- In Hospice.

Tali strutture lavorano come un'unità che guarda al Territorio e che lavora, anche fisicamente, con i Medici di famiglia, con i Distretti e che riceve i pazienti dimessi dalle Medicina Interne o dagli altri reparti.

Questa Unità ha grande valenza in un momento come questo, in cui i tentativi e gli sforzi maggiori sono nella direzione di riempire di contenuti il territorio.

Questa Unità può anche essere sede principale di screening per SC de novo, in grado di fornire specifiche competenze, ma anche dotazioni tecnologiche rilevanti (es. ECG) per intervenire in maniera coordinata nel PDTA ed in collegamento funzionale con l'Ospedale.

Corrispondente: Giuseppe Campagna.

E-mail: campagna.casa@libero.it

Articolo pubblicato secondo la Creative Commons Attribution

NonCommercial 4.0 License (CC BY-NC 4.0).

${ }^{\circ}$ Copyright: the Author(s), 2021

Licensee PAGEPress, Italy

QUADERNI - Italian Journal of Medicine 2021; 9(5):e8
Gli Ambulatori Ospedalieri per SC si collocano in Ospedali dotati di degenza ordinaria. Sono rivolti sia a pazienti stabili che a pazienti con SC di nuova insorgenza e/o recentemente instabilizzato.

Rispetto all'Ambulatorio territoriale, quello ospedaliero assicura il completamento dell'iter diagnostico-terapeutico del paziente e l'esecuzione di procedure anche di tipo interventistico con l'utilizzo dei PAC e/o dei Day Hospital. Questo Ambulatorio è collocato in UUOO Ospedaliere con competenza nella gestione di SC acuto.

L'Unità Scompenso e l'Ambulatorio Ospedaliero per le Cure del Paziente Avanzato sono collocati in Ospedali dotati di UUOO di Medicina Interna, Geriatria, Medicina d'Urgenza, Rianimazione e Cardiologia con degenza ordinaria, UTIC, laboratorio di emodinamica operativo 24 ore al giorno, 7 giorni su 7, elettrofisiologia e cardiochirurgia, con programma integrato medico-chirurgico per il trattamento di SC Avanzato e in casi selezionati il programma VAD/TCs.

Qui afferiscono i pazienti a più elevata complessità di gestione:

- Pazienti ad alto rischio di riacutizzazione.

- Pazienti in carico all'Ambulatorio Cardiologico Ospedaliero di prossimità ma bisognosi di valutazioni diagnostiche e trattamenti terapeutici non disponibili per quell'Ambulatorio.

- Pazienti che necessitano di procedure.

- Pazienti con scompenso cardiaco avanzato.

- Pazienti con scompenso cardiaco avanzato candidabili a terapia sostitutiva cardiaca.

Nel costruire la rete bisogna definire i bacini di utenza nel Comune/Provincia/Regione, individuare la Medicina Territoriale che si occupi di SC, definire i Centri di SC e gli ospedali dotati di Centro per SC Avanzato e dotati di Cardiochirurgie. E' necessario inoltre definire le Strutture Riabilitative Cardiologiche, organizzare il fine vita e le cure palliative, nonchè individuare il Responsabile aziendale del PDTA per lo SC.

L'architettura della rete è policentrica e pluri-gravitazionale e deve farsi carico sia dell'acuzie che della cronicità sull'intero spettro della storia clinica, dalla prima diagnosi alle fasi terminali della malattia.

Nell'architettura della rete, i Centri Scompenso di prossimità, di riferimento e con programma di terapia sostitutiva cardiaca, rappresentano l'offerta di prestazioni specialistiche e super-specialistiche. Nell'architettura della rete, la distribuzione dei centri scompenso 
e la loro tipologia potrà variare in base alla distribuzione geografica delle strutture sanitarie e, in rapporto a tale distribuzione, andranno identificate le prestazioni offerte dai diversi centri e i relativi percorsi ambulatoriali e di ricovero.

La rete si pone in modo aperto, pronta ad interagire con le diverse competenze plurispecialistiche, offrendo piena collaborazione con le strutture di degenza che accolgono i pazienti ospedalizzati e con le strutture territoriali.

E' infatti auspicabile che un paziente stabilizzato dopo aver eseguito, ad esempio, una procedura interventistica presso un Centro Ospedaliero possa tornare ad essere seguito dal Centro Territoriale, prevalentemente in regime ambulatoriale ed in sinergia con la Medicina Generale. 


\section{LINEE GUIDA PER GLI AUTORI}

I Quaderni dell 'Italian Journal of Medicine (Quaderni ITJM), costituiscono una collana supplementare solo online annessa alla rivista Italian Journal of Medicine contenente lavori solo in lingua italiana .

I Quaderni ITJM pubblicano:

- Monografie ad hoc individuate dal Presidente FADOI, dal Consiglio Direttivo, dal Board Scientifico o dall'Editor in Chief dell'Italian Journal of Medicine, in funzione del contesto scientifico-istituzionale attuale.

- Monografie ad hoc su temi di particolare rilevanza scientifica a cura della Commissione FADOI Giovani.

- Traduzioni in italiano di alcuni lavori pubblicati sui numeri standard dell'Italian Journal of Medicine, di particolare interesse per la comunità scientifica.

\section{STESURA DEI LAVORI}

I lavori dovranno essere redatti in modo conforme alle linee guida sotto riportate:

- I manoscritti devono essere scritti interamente in lingua italiana, su documento di Word, con carattere Times New Roman/Arial, dimensione 12, formato A4, interlinea doppia $\mathrm{e}$ margini $2,54 \mathrm{~cm}$.

Parole totali: $\max$ 4000; Sommario/Abstract: $\max 250$ parole: Bibliografia: $\min 40$ voci; Tabelle e Figure: $3 / 5$ totali (le tabelle non devono superare n. 1 pagina del documento in Word).

- La strutturazione del contenuto deve attenersi agli standard internazionali per la Rassegna (Review): i) Abstract riassuntivo dell'intero lavoro; ii) Introduzione al tema trattato; iii) Criteri e strumenti di ricerca (criteri di inclusione/esclusione, banche dati consultate, ...); iv) i successivi paragrafi devono illustrare le più recenti scoperte scientifiche nel settore; v) Conclusioni; vi) Bibliografia.

- La prima pagina deve riportare: i) titolo (in stampatello minuscolo), senza acronimi; ii) nome e cognome per esteso di ciascun autore; iii) affiliazione(i) di ciascun autore, numerate con numeri arabi; iv) eventuali ringraziamenti; v) nome e indirizzo postale completi dell'autore corrispondente, corredati da telefono, fax, e-mail; vi) da 3 a 5 parole chiave, separate da virgola. La seconda pagina può riportare: i) contributi degli autori, e.g. informazioni relative a contributi sostanziali delle persone coinvolte nello studio (http://www.icmje.org/\#author); ii) dichiarazione relativa a potenziali conflitti d'interesse; iii) ulteriori informazioni (e.g. fondi, esposizioni durante conferenze...).

- In caso di utilizzo di tabelle, queste devono essere tutte numerate con numeri arabi e citate nel testo in ordine consecutivo (e.g. NON nominare le tabelle come Tabella $1 \mathrm{~A}, 1 \mathrm{~B}, \ldots$ o $1.0,1.1, \ldots)$. Le tabelle devono essere presentate in formato editabile. Ciascuna tabella deve essere corredata da una breve didascalia; in caso di abbreviazioni, riportare una nota a piè di CIASCUNA tabella che spieghi TUTTE le abbreviazioni presenti in ognuna.

- In caso di utilizzo di figure, queste devono essere inviate in formato tiff o .jpg, allegate al manoscritto in singoli files, secondo le seguenti specifiche:

i) a colori (salvate in modalità CMYK): minimo 300 dpi di risoluzione;

ii) in bianco e nero: minimo 600 dpi di risoluzione;

iii) minimo $17,5 \mathrm{~cm}$ di larghezza.

Ciascuna figura deve essere corredata da una breve didascalia.

NB: In caso di Tabelle/Figure riprese e/o modificate da altri lavori già pubblicati, sarà cura degli autori accertarsi se tali materiali siano o meno coperti da copyright e procurarsi i permessi necessari per la riproduzione. Tali permessi dovranno essere allegati alla versione definitiva del lavoro. L'ufficio editoriale si riserva la facoltà di rimuovere Tabelle/Figure coperte da copyright, se sprovviste dei necessari permessi.

- In caso di utilizzo di abbreviazioni, la prima volta che esse sono citate è necessario scrivere per esteso la definizione+abbreviazione tra parentesi tonde [e.g. risonanza magnetica $(\mathrm{RMN})]$, a seguire si dovrà riportare solo l'abbreviazione (unica eccezione: nei titoli e nelle didascalie di tabelle e figure NON si utilizzano abbreviazioni).

\section{BIBLIOGRAFIA}

Le voci bibliografiche devono essere formattate secondo lo stile Vancouver.

Nella sezione Bibliografia, le voci bibliografiche devono essere numerate consecutivamente nell'ordine in cui appaiono per la prima volta nel testo (NON in ordine alfabetico) e, nel testo, devono essere indicate con numeri arabi in apice. Voci bibliografiche riferite a comunicazioni personali o dati non pubblicati devono essere incorporate nel testo e NON inserite tra le voci numerate [e.g. (Wright 2011, dati non pubblicati) o (Wright 2011, comunicazione personale)]. Le voci bibliografiche nella sezione Bibliografia devono tassativamente essere preparate come segue:

i) più di 3 autori, citare 3 autori, et al. Se il lavoro contiene solo 4 autori, citarli tutti e 4 ;

ii) titolo del lavoro in stampatello minuscolo;

iii) nome della rivista, senza punti, abbreviato secondo gli standard internazionali; in caso di dubbi sulla corretta abbreviazione, fare riferimento ai seguenti siti:

a. ISI Journal Abbreviations Index (http://library.caltech.edu/ reference/abbreviations/);

b. Biological Journals and Abbreviations (http://home.ncifcrf. gov/research/bja/);

c. Medline List of Journal Titles (ftp://ftp.ncbi.nih.gov/pubmed/ J_Medline.txt);

iv) inserire l'anno di pubblicazione subito dopo il nome della rivista, seguito da punto e virgola;

v) NON inserire giorno o mese di pubblicazione;

vi) citare solo il volume, seguito dai due punti (NON citare il fascicolo tra parentesi);

vii) abbreviare le pagine, e.g. 351-8.

Per accertarsi di aver correttamente formattato le voci bibliografiche, confrontarle con le citazioni in PubMed (http://www.ncbi. nlm.nih.gov/pubmed).

Esempi (prestare attenzione anche alla punteggiatura):

Articolo standard su Rivista

Halpern SD, Ubel PA, Caplan AL. Solid-organ transplantation in HIV-infected patients. N Engl J Med 2002;347:284-7.

Proceedings

Christensen S, Oppacher F. An analysis of Koza's computational effort statistic for genetic programming. In: Foster JA, Lutton E, Miller J, Ryan C, Tettamanzi AG, eds. Genetic programming. EuroGP 2002: Proceedings of the 5th European Conference on Genetic Programming, 2002 Apr 3-5, Kinsdale, Ireland. Berlin: Springer; 2002. pp 182-91.

Articoli i cui autori sono Organizzazioni

Diabetes Prevention Program Research Group. Hypertension, insulin, and proinsulin in participants with impaired glucose tolerance. Hypertension 2002;40:679-86.

Libri

Murray PR, Rosenthal KS, Kobayashi GS, Pfaller MA. Medical microbiology. 4th ed. St. Louis, MO: Mosby; 2002. (CITAZIONE DEL LIBRO INTERO)

Meltzer PS, Kallioniemi A, Trent JM. Chromosome alterations in human solid tumors. In: Vogelstein B, Kinzler KW, eds. The genetic basis of human cancer. New York, NY: McGraw-Hill; 2002. pp 93113. (CITAZIONE DI UN CAPITOLO)

\section{MODALITÀ D'INVIO DEI LAVORI}

Monografie

Gli Autori dovranno fare riferimento all'Editor-in-Chief o alle persone da lui designate nelle lettere di invito a scrivere gli articoli programmati.

Per gli articoli inviati da giovani internisti, gli Autori dovranno fare riferimento al Dr. Michele Meschi (e-mail: mmeschi@ausl.pr.it)e agli eventuali altri referenti da lei designati, nelle prime fasi di stesura dei manoscritti, revisioni e correzioni. 
Il Dr. Meschi raccoglierà poi le versioni definitive dei lavori di ciascuna monografia e provvederà all'invio di tutti i materiali all'ufficio editoriale.

I lavori solo nella loro versione definitiva e approvata dalla Commissione FADOI Giovani dovranno pervenire all'ufficio editoriale già pronti per l'impaginazione e immediata pubblicazione (già corredati da eventuali permessi per la riproduzione di tabelle e immagini redatti secondo le presenti linee guida).

Traduzioni

Previo invito dell'Editor-in-Chief, gli Autori dovranno far pervenire all'ufficio editoriale la versione tradotta in italiano, al seguente indirizzo e-mail: paola.granata@pagepress.org

Il file in formato Word dovrà essere formattato secondo gli standard editoriali della rivista ufficiale ed essere già pronto per impagina-

STAFF EDITORIALE

Paola Granata, Journal Manager

paola.granata@pagepress.org

Claudia Castellano, Production Editor

Tiziano Taccini, Technical Support

\section{PUBBLICATO DA}

PAGEPress Publications

via A. Cavagna Sangiuliani 5

27100 Pavia, Italy

T. +39.0382 .1549020$

F: +39.0382 .1727454$

\section{press}

www.pagepress.org info@pagepress.org zione e immediata pubblicazione (corredato da eventuali permessi per la riproduzione di tabelle e immagini).

Si prega di inviare le eventuali tabelle in formato editabile e le figure in alta definizione secondo gli standard sopra riportati.

\section{NOTA PER GLI AUTORI}

I lavori pubblicati sui Quaderni ITJM non verranno indicizzati, ma saranno liberamente disponibili in un'apposita sezione del sito FADOI (http://www.fadoi.org/) e della rivista ufficiale.

Gli Autori i cui lavori siano accettati per la pubblicazione sui Quaderni ITJM $e$ che fossero interessati a vederli pubblicati anche sulla rivista ufficiale, dovranno sottomettere attraverso il sito dell'ITJM (www.italjmed.org) la versione (già tradotta) in inglese e redatta in modo conforme alle linee guida della rivista; seguiranno poi la procedura di selezione tramite peer review e, se accettati, saranno inseriti nel piano editoriale standard.

\section{QUADERNI - ITALIAN JOURNAL OF MEDICINE}

Tutti gli articoli pubblicati sui QUADERNI - Italian Journal of Medicine sono redatti sotto la responsabilità degli Autori. La pubblicazione o la ristampa degli articoli della rivista deve essere autorizzata per iscritto dall'editore. Ai sensi dell'art. 13 del D.Lgs 196/03, i dati di tutti i lettori saranno trattati sia manualmente, sia con strumenti informatici e saranno utilizzati per l'invio di questa e di altre pubblicazioni e di materiale informativo e promozionale. Le modalità di trattamento saranno conformi a quanto previsto dall'art. 11 del D.Lgs 196/03. I dati potranno essere comunicati a soggetti con i quali PAGEPress intrattiene rapporti contrattuali necessari per l'invio delle copie della rivista. Il titolare del trattamento dei dati è PAGEPress Srl, via A. Cavagna Sangiuliani 5 - 27100 Pavia, al quale il lettore si potrà rivolgere per chiedere l'aggiornamento, l'integrazione, la cancellazione e ogni altra operazione di cui all'art. 7 del D.Lgs 196/03.

https://www.italjmed.org/index.php/ijm/quad 


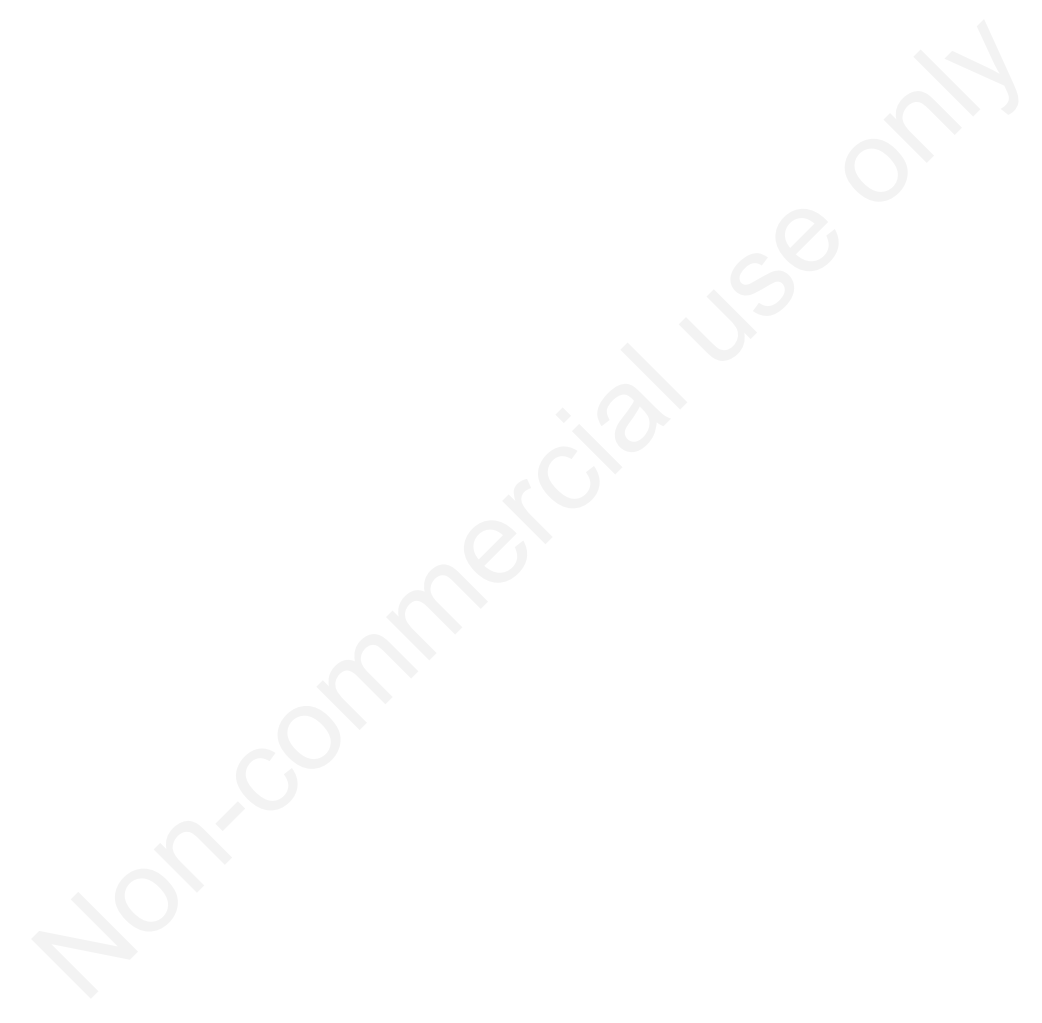


